\title{
EXTRACTION OF DEMS AND ORTHOIMAGES FROM ARCHIVE AERIAL IMAGERY TO SUPPORT PROJECT PLANNING IN CIVIL ENGINEERING
}

\author{
M. Cogliati a,1, E. Tonelli ${ }^{\text {b }}$, D Battaglia ${ }^{\text {c }}$, M. Scaioni a,b \\ a Polo Territoriale di Lecco, Politecnico di Milano, via G. Previati 1/c, 23900 Lecco, Italy - marco2.cogliati@mail.polimi.it \\ b Dept. of Architecture, Built Environment and Construction Engineering (DABC), Politecnico di Milano, via Ponzio 31, 20133 Italy \\ (marco.scaioni, eliana.tonelli)@polimi.it \\ c Georisk, via Stelvio 20, Triuggio (MB), Italy
}

Commission V, WG V/7

KEY WORDS: Aerial photogrammetry, Analogue, Archive photos, Civil engineering, Low-cost, Project planning

\begin{abstract}
:
Archive aerial photos represent a valuable heritage to provide information about land content and topography in the past years. Today, the availability of low-cost and open-source solutions for photogrammetric processing of close-range and drone images offers the chance to provide outputs such as DEM's and orthoimages in easy way. This paper is aimed at demonstrating somehow and to which level of accuracy digitized archive aerial photos may be used within a such kind of low-cost software (Agisoft Photoscan Professional ${ }^{\circledR}$ ) to generate photogrammetric outputs. Different steps of the photogrammetric processing workflow are presented and discussed. The main conclusion is that this procedure may come to provide some final products, which however do not feature the high accuracy and resolution that may be obtained using high-end photogrammetric software packages specifically designed for aerial survey projects. In the last part a case study is presented about the use of four-epoch archive of aerial images to analyze the area where a tunnel has to be excavated.
\end{abstract}

\section{INTRODUCTION}

Since the beginning of its history, photogrammetry has been featuring two distinctive properties with respect to other surveying techniques. The first one is the huge metric and semantic information content that may be extracted from images, depending on photo scale and photo quality. The second is given by the chance to derive new pieces of information at any moment, unlike standard geodetic or GNSS techniques, which may only record those elementary geometric features (points, polylines, areas) 'selected' during surveying operations. This latest property allows to retrieve information about topography, buildings and land use during past decades, thanks to the availability of existing large archives of aerial photos. Consequently, the content that is not already mapped in cartography but dated back after the first satellite image cover, may be also investigated.

First analogue satellite imagery were collected in the '60s, when a few data sets of images for intelligence surveillance were captured and, after decades, declassified and made available to the public (Kim et al., 2007; Li et al., 2017). At the beginning of the '70s the era of spaceborne imaging sensors started and has impressively developed up until today. Areas not covered by satellite images or where these do not feature a sufficient resolution, may have been depicted during analogue aerial photogrammetry missions accomplished for scientific investigation over specific areas (e.g., in Antarctica - see Kunz et al., 2012), or for topographic and cadastral mapping purpose. The second class of missions represents the large majority of available data sets.

The aim of retrospective analysis is clearly evident in the case of scientific or historical studies. In such cases, the change (or stability) that may be understood from the comparison of multi- temporal archive images may offer important outputs for comprehending undergoing processes (see, for example, Walstra et al., 2007). On the other hand, there are several applications in forensics, urban planning and civil engineering where archive photos may also provide a relevant support. For example, in many legal controversies some evidence about how a terrain was used in the past or about which was the condition of a building is needed. The interpretation of archive photos may help come up with a solution in many of these cases. In addition, photogrammetry also permits to derive metric 3D reconstructions if at least a stereoscopic pair of images is available, see Kraus (2008). A stereo pair is made up of two images with partial overlap (approx. between 60\%-80\%) recorded from camera positions shifted along the same flight-line with tiny relative orientation angles. Consequently, not only may be the 2D image content investigated, but also the extraction of retrospective surface models (DSM) is achievable. This option opens the scene to an even wider range of technical applications.

An important consideration has to be made now to introduce the specific contribution of this paper. The extraction of 3D metric information from airborne photos was a task to be operated only by specialists as far as photogrammetry has not entered yet the digital era, i.e., across the '80s and '90s (see Ackermann, 1991; Grün, 2012). Stereo-plotting required expensive analytical instruments, whose operation needed highly-specialized technicians. But also in the first stage of the digital era, analogue photos were transformed into digital support and then processed with specific digital photogrammetric workstations (Dowman et al., 1992). Due to the cost of computing machines able to run the photogrammetric software and the cost of the software itself, the popularity of photogrammetry to extract 3D information was still far-away to become accessible to a wide range of people.

\footnotetext{
${ }^{1}$ Corresponding author
} 
Progresses in computers and the integration of photogrammetric and computer vision methods have contributed to create the current state-of-the-art of modern photogrammetry. In parallel with costly systems for industrial map production, an impressive number of low-cost or even open-source software packages have started to spread out during the last decade. In addition, these packages offer to non-expert people the chance to derive 3D models and orthoimages from images collected using multi-platform cameras: from ground stations (close-range or terrestrial photogrammetry, see Luhmann et al., 2014), from unmanned aerial systems (UAS - see Colomina \& Molina, 2014), and from manned aircrafts equipped with large-format airborne digital cameras (Toth \& Jozkow, 2016).

The use with digitized old aerial analogue photos is not directly encompassed in the most low-cost photogrammetric systems, which have been designed to work mainly with closerange and UAS projects. As it can be seen in Figure 1, an analogue photo has a framework reporting data about acquisition time, adopted camera and lens, temperature, and 4-8 fiducial marks (FM - see Kraus, 2008). In particular, FM's are used for correcting residual film distortions that may have developed during the conservation of the analogue support, for example. The typical size of these distortions may be approximately few tens of micrometres after a few decades, depending on somehow the film has been archived. FM's were concurrently used for establishing the photo reference system, which is centred in the middle of the frame.

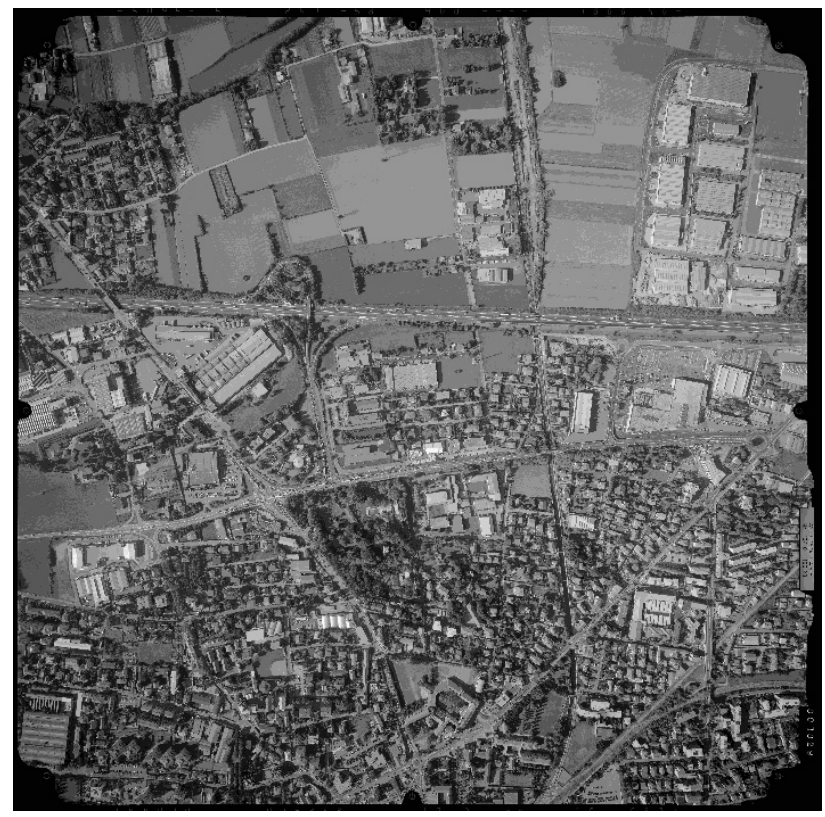

Figure 1. Example of an analogue photo with eight FM's captured with a Wild RC30 airborne metric camera. This image is included in 'Test block' described at the beginning of Section 3 .

The purpose of this paper is to show how the processing of digitized analogue photos retrieved from the archives may be carried out with low-cost photogrammetric software packages to produce DSM's and orthoimages. The quality that may be obtained is analysed and discussed in this paper, so that the user may understand which kinds of applications are affordable using this approach. Here the popular low-cost software Agisoft Photoscan Professional ${ }^{\circledR}$ has been considered. The adopted technical workflow (Sect. 2) is proposed along with the presentation of a case study in Section 3. This consists in the use of archive photos to investigate an area located in a mountain valley where a new road tunnel had to be constructed.

\section{METHOD}

The method for deriving mapping products (DSM's and orthoimages) from digitized archive photos has been based on the application of low-cost photogrammetric software package Agisoft Photoscan Professional $^{\circledR}$ (APP) ver. 1.2.4 (www.agisoft.com). This software is able to work in fully automatic way using a Structure-from-Motion (SfM) approach (Hartley \& Zisserman, 2006; Barazzetti et al., 2011a) for image orientation, followed by dense image matching operated in a multi-view stereo fashion (Forlani et al., 2015). The only manual task is the measurement of some ground control points (GCP) necessary for establishing the geodetic datum to mitigate the instability in successive bundle block adjustment (BBA), see Verhoeven et al. (2015). In general, APP is used for non-metric digital cameras, which do not feature a specific frame on borders (see Fig. 1). Even though a pre-calibrated camera may be used, during BBA stage, APP may compute the unknown camera calibration parameters using a self-calibration approach based on tie points extracted during SfM (Barazzetti et al., 2011b). When dealing with digitized photos from airborne metric cameras, some considerations on how to deal with the interior orientation in APP should done (see Subsect. 2.1). In the following subsections the successive steps are described: SfM for image exterior orientation (Subsect. 2.2), GCP measurement (Subsect. 2.3), assessment of the orientation quality (Subsect. 2.4), dense image matching (Subsect. 2.5), and orthophoto generation (Subsect. 2.6).

A small block ('Test block') made up of four photos along the same strip has been used to demonstrate somehow different options available in APP may affect the completeness and the accuracy of photogrammetric outputs. Figure 1 depicts one of the photos from this block, flown over a small town in northern Italy, including both urbanized and rural land cover. The flight was operated in 2008 using a high-quality airborne metric camera Wild RC30, equipped with a 'normal' lens (focal lens 152.28 $\mathrm{mm}$ ). The average photo scale was 1:8,350 with a ground sample distance (GSD) of approximately $10 \mathrm{~cm}$. Colour analogue photos were transformed into grayscale digital images (size 16,752 x 16,644 pixels, pixel size $14 \mu \mathrm{m}$ ) using a photogrammetric scanner. Four GNSS GCP's were available for precise georeferencing in UTM mapping grid. Automatic masking has been applied in APP to avoid the extraction of points on the outer frame. This function has successfully managed to exclude the outer frame from successive processing tasks.

\subsection{Inner orientation}

The main problem here is to reconduct the interior orientation (IO) of an analogue camera for which the calibration certificate may not be available, to the IO-model normally adopted in closerange photogrammetry, i.e., the Brown's model (see Luhmann et al., 2016). For the applications discussed in this paper, generally recourse is made to small blocks of aerial photos imaged by the same camera. Thus, the estimate of IO parameters on the basis of self-calibration during BBA is not fully reliable due to the tiny number of employed photos. In the following, an analysis of individual parameters that have to be reconstructed in the Brown's model is reported for the general case of an analogue photo captured from a metric camera. 
The principal distance $c$ can be read on the photo frame, even though the reported value may slightly differ from the one in the calibration certificate matching to data acquisition time exactly. Since all photos in a block typically share the same value for $c$, it may be adjusted when computing the absolute orientation, i.e., when GCP's are included: a variation of the estimated elevations of perspective centre of each photo may compensate an error in the determination of $c$. The same approach may be used for the principal point offset $\left(x_{0}, y_{0}\right)$. If the original analogue photos have been manually placed on the scanner plate, it may result that the image reference system has a different origin and a small rotation in each image. This problem may be neglected in the case scanning has been done automatically from the film roll. To correct the presence of large rotations and to transform all images in the same condition, the following procedure may be worth to be applied:

1. by using a software for image editing, the photo frame can be rotated to become parallel to the image frame. The existing rotation may be evaluated by considering the image coordinates ( $r, c$ - row and column) of the centres of two FM's at the corners of a photo on the left (or right) side, see Fig. 1 . The rotation angle $\theta$ is computed using the following formula:

$$
\theta=\operatorname{atan} \frac{c_{2}-c_{1}}{r_{2}-r_{1}}
$$

where $\left(r_{1}, c_{1}\right)$ and $\left(r_{2}, c_{2}\right)$ are image coordinates of the centres of FM's at upper and lower corners, respectively.

2. After compensating for the rotation $\theta$, the image may be resized by cropping the actual photo area at the inner borders of the frame. After this operation, both rows and columns should be the same: if this condition is not verified, the presence of distortion due to scanning or severe film deformations should be investigated.

This processing scheme may be repeated independently per each image. Alternatively, the correction process described at point 1 may be applied to one photo only. Then other images may be rectified to this one using fiducial marks as control points. This method also allows to check out whether all the images are geometrically identical.

The Brown's model entails three different types of distortions: (i) radial-symmetric distortion; (ii) decentering distortion; and (iii) affinity and shear. In theory, the use of accurately calibrated metric cameras equipped with 'normal' lens $(c \cong 150 \mathrm{~mm}$ ) should provide images where only a minimal radialsymmetric distortion exists. The effect of this distortion may be evaluated in the order of a few micrometres in the border region of the image, then negligible in many applications.

The presence of other types of distortions may be motivated by deformations occurred during scanning (see Baltsavias, 1994), in particular those inherent to affinity and shear. The compensation of this latest type of distortion may be carried out during self-calibration BBA, but limited to blocks made up of at least 4-5 photos. In addition, affinity and shear distortion may model the effects of two different linear scaling along both orthogonal directions, but does not account for non-linear scaling that may occur during scanning of analogue films when using non-photogrammetric scanners.

\subsection{Structure-from-Motion}

The SfM technique is implemented in APP to compute the exterior orientation (EO) of a block of close-range images. It is out of the scope of this paper to review SfM theoretical aspects nor to go into details of how SfM is implemented in APP, where it is termed 'Image alignment'. Briefly the aim of this subsection is to highlight some aspects to be considered when dealing with a small block of digitized analogue photos in APP, such as the 'Test block.'

The most important input parameter to set up is the level of 'Accuracy' that controls the precision of tie-point (TP) matching. At the highest level, corresponding TP's are automatically measured at the native resolution of the scanned images. Thus, a longer processing time may be needed, especially when the size of the block is really huge (hundreds of images). The lowest level is useful to do a fast orientation for preliminary check out, since it is operated on subsampled imagery. When the number of analogue archive photos is small, the suggestion is to compute the EO at 'Highest Accuracy' level, in order to have a solid metric basis for successive dense matching, but with a processing time limited to a few minutes. A chance to save time is given by the option to set up an upper bound on the number of key-points to be extracted, which are the candidates for TP selection, and the maximum number of total TP's. In general, 100 well-distributed TP's per image are more than enough to obtain a stable EO. This set of extracted TP's makes the so called 'Sparse point cloud' to be used as approximate model for dense matching (Previtali et al., 2014). Further considerations are reported in Subsection 2.4. Since TP's may be also erroneously extracted on the external frame of the photo, a mask may be applied to exclude that region from active processing.

\subsection{Ground control points}

In small blocks, the aim of GCP's is merely to establish the datum where the final outputs are wanted. From a mathematical point of view, a minimum set of three GCP's is needed to remove the rank deficiency in EO, corresponding to a seven-parameter 3D similarity transformation (see Kraus, 2008). A larger number of GCP's may be useful to rise up the local redundancy and then the controllability against blunders (Kraus, 1987). Six GCP's may be a suggested number for a stereo pair, nine GCP's in the case of three photos, 12 for four photos and so on. The real problem in the case of old archive photos is how to derive the reference ground coordinates of GCP's. In fact, the objects in the photo may have dramatically changed from the time when the photo was captured and the present time. A typical solution to derive GCP's is to obtain their horizontal position from orthoimages and the elevation from a Digital Terrain Model (DTM). In Figure 2 the position of four GCP's measured in the 'Test block' is shown together with the extracted TP's.

\subsection{Assessment of the exterior orientation quality}

The assessment of the quality of EO may be based on results displayed in the 'Reference' pane of APP and the report that may be generated after processing (menu 'File -> Generate Report'). A first analysis should concern TP number and distribution. Aspects that should be investigated are:

1. Average number and distribution of TP's: a minimum number of well distributed TP's all over the overlap region(s) of the images is necessary to grant a correct estimation of EO parameters; 


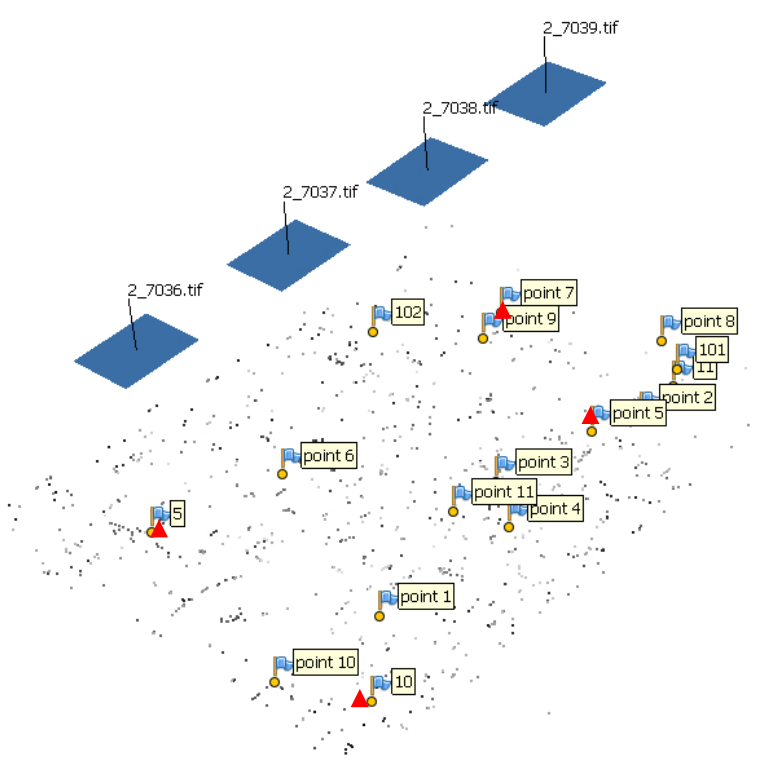

Figure 2. Positions of points and images in the 'Test block:' TP's (points), GCP's (red triangles), control points (yellow points with blue flag); and images (rectangles).

1. RMS (Root mean squared) of TP reprojection errors in each image: it gives and a metrics on how well the computed 3D coordinates of TP's in the model space may fit with corresponding 2D image coordinates after reprojection on an image. In general, a subpixel average value should be preferably obtained;

2. Average number of rays per TP: it may be obtained as:

$$
\text { avg_rays_TP }=\frac{\# \text { projections }}{\# T P}
$$

This value is important only in the case of blocks made up of more than two images (in such a case, avg_rays_TP=2), where the presence of manifold TP's that have been measured on more than two images may improve the inner reliability of the observations and help the detectability of blunders (see Kraus, 1987); and

4. RMSE of GCP residuals: it expresses how well the EO fits with GCP coordinates. This figure may be assumed as measure of the block accuracy if the number of GCP is high (e.g., more than six).

The measurement of additional check points (ChP) may give an independent evaluation of the block accuracy, even in the case few GCP's have been fixed. ChP's may come from a subset of GCP's that have not been actively used for computing the EO. In Table 1 the comparison of performances obtained during the computation of the EO of the 'Test block' is reported.

In order to figure out the discrepancies that can be obtained in terms of object point coordinates, a set of 11 control points (CP) have been manually measured on the images of this block (see Fig. 2). Their object coordinates have been worked out on the basis of the EO's corresponding to different configurations in Table 1 . The absolute discrepancies between 3D coordinates of these CP's are shown in Table 2. As it may be seen from these findings, the results obtained with different EO parameters corresponding to the 'Accuracy' levels are quite uneven, especially on the elevations.

\begin{tabular}{|l|l|c|c|c|c|c|}
\hline 'Accuracy' level & $\begin{array}{c}\text { Very } \\
\text { low }\end{array}$ & Low & Med & High & $\begin{array}{c}\text { High- } \\
\text { est }\end{array}$ \\
\hline \#TP's & 1103 & 1549 & 2197 & 3114 & 3329 \\
\hline Avg. \#rays/point & 2.07 & 2.06 & 2.07 & 2.10 & 2.15 \\
\hline $\begin{array}{l}\text { RMSE } \\
\text { image reproj. } \\
\text { [pix] }\end{array}$ & $\mathbf{T P}$ & 1.2 & 1.0 & 0.6 & 0.4 & 0.3 \\
\hline $\begin{array}{l}\text { RMSE } \\
\text { GCP [cm] }\end{array}$ & $\mathbf{G C P}$ & 1.1 & 1.2 & 0.9 & 0.8 & 0.8 \\
\cline { 2 - 7 } & $\boldsymbol{H}$ & 0.7 & 0.8 & 0.9 & 0.4 & 0.4 \\
\cline { 2 - 7 } & $\mathbf{3 D}$ & 1.3 & 1.6 & 1.6 & 0.8 & 0.9 \\
\hline \multicolumn{2}{|l|}{ Time for EO } & $1^{\prime 2} 20^{\prime \prime}$ & $11^{\prime} 13^{\prime \prime}$ & $1^{\prime} 33^{\prime \prime}$ & $2^{\prime} 18^{\prime \prime}$ & $6^{\prime} 16^{\prime \prime}$ \\
\hline
\end{tabular}

Table 1. Statistic on the computation of EO of 'Test block' using different configurations for EO ('Image alignment') in APP.

\begin{tabular}{|l|c|c|c|}
\hline EO ‘Accuracy’ & E-N [m] & H [m] & 3D [m] \\
\hline High-Highest & 0.32 & 0.25 & 0.41 \\
\hline Medium-Highest & 1.99 & 19.35 & 19.45 \\
\hline Low-Highest & 2.99 & 15.63 & 15.91 \\
\hline Very low- Highest & 4.65 & 9.72 & 10.77 \\
\hline
\end{tabular}

Table 2. RMSE of coordinates of 11 CP's computed on the basis the EO parameters obtained from processing at 'Highest Accuracy' level (assumed as reference) and with other 'Accuracy' levels.

\subsection{Dense image matching}

The reconstruction of the topographic surface usually requires the densification of the 'Sparse point cloud' previously obtained. This is the aim of dense image matching phase (Forlani et al., 2015). In APP this stage is carried out on the basis of a state-ofthe-art multi-view stereo (MVS) dense image matching technique. It is based on the IO/EO computed during previous steps, and the definition of the area where a 'Dense point cloud' has to be generated. This task may be done in the photo space, through drawing a mask to define a region-of-interest, or in object space by using a moveable control box. Two main input parameters have to be set up before processing. 'Quality' parameter controls the resolution of the 'Dense point cloud,' using the same levels as in the image EO stage. From the experience achieved in processing of several digitized analogue photos, it is difficult to obtain an acceptable result at the highest 'Quality' level, especially over urbanized areas. The 'Medium' level may be the maximum to be used with this kind of photos, unlike in close-range projects where a higher level of 'Quality' may be chosen. The large size of digitized analogue photos may be responsible for this failure. A second input parameter is 'Depth filtering,' which also is organized on levels from 'Disabled' to 'Aggressive.' This filter has a surface smoothing effect to be applied with noisy images or where high-frequency details are abundant.

In Table 3 the comparison of results obtained for dense matching phase are reported. Two configurations for the EO were fixed to compute the 'Dense point cloud': 'Medium' and 'Very high.'

\subsection{Digital Elevation Model and orthoimage generation}

The 'Dense point cloud' is an intermediate product of photogrammetric processing, which has to be further elaborated to obtain some final outputs necessary in applications, mainly the Digital Elevation Model (DEM) and the orthoimages. 


\begin{tabular}{|c|c|c|c|c|c|c|}
\hline & \multirow{2}{*}{$\begin{array}{c}\text { Adopted } \\
\text { 'Accuracy' } \\
\text { of EO }\end{array}$} & \multicolumn{5}{|c|}{ ‘Quality' level for dense matching } \\
\hline & & $\begin{array}{l}\text { Very } \\
\text { low }\end{array}$ & Low & Med & High & $\begin{array}{l}\text { Very } \\
\text { High }\end{array}$ \\
\hline \multirow{2}{*}{$\begin{array}{l}\text { Time for } \\
\text { dense } \\
\text { matching }\end{array}$} & Medium & $45^{\prime \prime}$ & $48^{\prime \prime}$ & $22^{\prime} 13^{\prime \prime}$ & $19^{\prime} 56^{\prime \prime}$ & N.C. \\
\hline & Highest & $53^{\prime \prime}$ & $47^{\prime \prime}$ & $1^{\prime} 52^{\prime \prime}$ & $18^{\prime} 08^{\prime \prime}$ & N.C. \\
\hline \multirow{2}{*}{$\begin{array}{l}\text { \#points of } \\
\text { 'Dense } \\
\text { point } \\
\text { cloud' }\end{array}$} & Medium & $1.0 \mathrm{M}$ & $2.8 \mathrm{M}$ & $5.3 \mathrm{M}$ & $6.1 \mathrm{M}$ & N.C. \\
\hline & Highest & $1.0 \mathrm{M}$ & $2.8 \mathrm{M}$ & $5.3 \mathrm{M}$ & $6.1 \mathrm{M}$ & N.C. \\
\hline
\end{tabular}

Table 3. Statistic on the dense matching of 'Test block' using different configurations for 'Dense point cloud' generation in APP and two sets of computed EO parameters ('Medium' and 'Very High Accuracy').

2.6.1 Digital Elevation Model: When topographic models corresponding to different epochs have to be compared to detect changes, resampling of each raw point cloud in correspondence of the nodes of a common raster grid (DEM) is usually the most convenient solution (see Lindenbergh \& Pietrzk, 2015). This solution allows to compute surface changes by simply subtracting elevations at corresponding points to obtain the DoD (Difference of DEM’s).

The generation of a DEM in APP is based on resampling the 'Dense point cloud' in a rectangular user-defined region. The grid spacing is autonomously defined by the software depending on the actual resolution of 'Dense point cloud.' Contour lines, if needed, may be also generated from the DEM afterwards. Later on, DEM may be exported in common file formats for raster topographic data (including basic metadata for geo-referencing). At this stage, a new windowing and/or resampling may be applied to the DEM.

2.6.2 Orthoimage: An orthoimage is an orthographic projection where a DTM is mapped using oriented photos. In APP two alternative approaches may be applied for generating orthoimages: (1) by creating a textured mesh from the 'Dense point cloud'; or (2) by directly mapping the DEM. The option (2) has been selected in the case of aerial photos, since it follows the standard approach for generating orthoimages in this type of projects. Also with small photogrammetric blocks, the area to process is imaged in at least a couple of photos. Thus, the radiometric content of each pixel in the output orthoimage may be blended in different ways, for example, by the automatic selection of the photo closer to the nadiral position of each pixel. The final orthoimage may be exported in standard geo-referenced formats.

\section{THE CASE STUDY}

\subsection{Motivations}

The occasion to investigate a few data sets of archive analogue photos came up during the design stage of a road tunnel $(1,072 \mathrm{~m}$ long) to by-pass the village of Kiens/Chienes in Pustertal/Val Pusteria (South Tyrol, Italy). Up until today this village is crossed by the State Road SS49, which is the main way to connect villages and tourist resorts in the valley to the Bozen valley (Italy) on one side, and to the Eastern Tyrol (Austria) on the other end. This by-pass should reduce the volume of road traffic crossing the village of Kiens/Chienes and concurrently speed up the traffic on the SS49. The path of the new tunnel is shown in Figure 3: it follows the longitudinal axis of a road on surface, minimizing the impact only on a small number of existing buildings.
After the definitive approval of this project in 2010, the executive design could be started. A thorough historical analysis of the area interested by construction works was necessary to assess the presence of anthropogenic or natural processes that might have altered the local geotechnical properties of the ground. For example, it was necessary to understand whether some areas might have been filled with reported debris or might have been interested by hydrogeological hazards.

Since the main growth of the urbanization in this area has developed during the last 50 years, a decision was made to do a preliminary analysis of topographic and cadastral maps, as well as the available archive photos dating back to that time. It has to be highlighted that this analysis of existing or newly-produced topographic data did not replace geotechnical in-situ investigations, but it was afforded only to figure out the possible presence of unknown problems, to be better focused in a following stage with local reconnaissance.

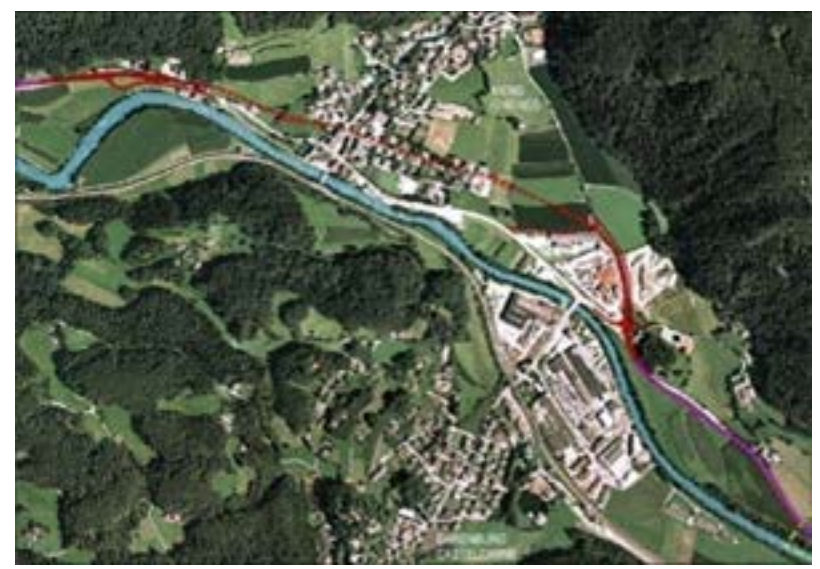

Figure 3. The longitudinal axis (red line) of the new tunnel to by-pass the village of Kiens/Chienes.

\subsection{Materials}

Four data sets from 1954 to 1995 were bought from IGM (Military Geographic Institute), which is the official national mapping agency in Italy. Each data set was made up of three analogue photos consecutively collected along the same flightline. The average longitudinal overlap ranged between $60 \%$ and $70 \%$. All photos were acquired using photogrammetric cameras equipped with 'normal' lens. No calibration certificates could be retrieved from IGM. The main features of all photos are displayed in Table 4.

Digital copies of these photos were obtained from original analogue versions using a non-photogrammetric scanner. A pixel size of $40 \mu \mathrm{m}$ was used for the output images. Consequently, in this project any possible geometric distortions introduced by scanning cannot be investigated (Baltsavias, 1994).

Average photo scales and relative flight heights were computed per each small block and reported in Table 4. The former could be obtained by considering two points identifiable in both the archive photos and in modern orthophotos covering the same area. The ratio between the distance on the photo and the corresponding distance on the orthophoto provided the photo scales (1:M). Then the average flight height was derived by using the proportion (see Kraus, 2008): 


\begin{tabular}{|c|c|c|c|c|c|}
\hline $\begin{array}{c}\text { Time } \\
\text { [DD/MM/ } \\
\text { YYYY] }\end{array}$ & $\begin{array}{c}\text { Strip } \\
\text { Id }\end{array}$ & $\begin{array}{c}\text { Photo } \\
\text { Id }\end{array}$ & $\begin{array}{c}\boldsymbol{c} \\
\text { [mm] }\end{array}$ & $\begin{array}{c}\text { Photo } \\
\text { scale } \\
\mathbf{1 : M}\end{array}$ & $\begin{array}{c}\text { Relative } \\
\text { flight height } \\
\text { Z } \text { [m] }\end{array}$ \\
\hline $01 / 09 / 1954$ & 24 & 3529 & 153.16 & $1: 47,775$ & 7317 \\
\hline $01 / 09 / 1954$ & 24 & 3530 & 153.16 & $1: 47,775$ & 7317 \\
\hline $01 / 09 / 1954$ & 24 & 3531 & 153.16 & $1: 47,775$ & 7317 \\
\hline $25 / 08 / 1959$ & 29 & 92 & 153.52 & $1: 27,834$ & 4273 \\
\hline $25 / 08 / 1959$ & 29 & 93 & 153.52 & $1: 27,834$ & 4273 \\
\hline $25 / 08 / 1959$ & 29 & 94 & 153.52 & $1: 27,834$ & 4273 \\
\hline $01 / 10 / 1986$ & 70 & 96 & 152.95 & $1: 28,916$ & 4423 \\
\hline $01 / 10 / 1986$ & 70 & 97 & 152.95 & $1: 28,916$ & 4423 \\
\hline $01 / 10 / 1986$ & 70 & 98 & 152.95 & $1: 28,916$ & 4423 \\
\hline $15 / 04 / 1995$ & 8 & 2873 & 152.73 & $1: 43,925$ & 6709 \\
\hline $15 / 04 / 1995$ & 8 & 2874 & 152.73 & $1: 43,925$ & 6709 \\
\hline $15 / 04 / 1995$ & 8 & 2875 & 152.73 & $1: 43,925$ & 6709 \\
\hline
\end{tabular}

Table 4. Properties of data sets of archive analogue photos.

$$
c: Z=1: M
$$

where $Z$ is the relative flight height with respect to ground.

In addition, five orthoimages were downloaded from Geoportale Nazionale Minambiente (www.pcn.minambiente.it/ GN/), dating back to: 1988, 1994, 2000, 2006, 2012. These data sets were used to derive GCP's to establish the geodetic datum of photogrammetric projects and to enrich the time series to analyse.

\subsection{Photogrammetric processing in APP}

A standard processing workflow was operated on the four data sets made up of digitized archive aerial photos. Photos were masked to avoid the interference from the outer frames and to limit editing. Image orientation of synchronous photos was conducted at 'High Accuracy' level using the SfM function ('Image alignment') implemented in APP. A set of GCP's was derived from existing geodata: planimetric GCP's were obtained from orthoimages, while altimetric GCP's from a LiDAR DTM. However, the residuals on GCP's have resulted quite large, due to the difficulty of recognizing the same points in the archive photos at relatively low resolution. Consequently, the decision of using only a minimum control has been made (i.e., three GCP's per photogrammetric block).

After the extraction of TP's and the measurement of GCP's on the images, a BBA was computed to estimate the IO and EO parameters. About the IO, the following coefficients of the Brown's model were estimated: c, $x_{0}, y_{0}$, two coefficients to compensate for radial symmetric distortion $\left(k_{1}, k_{2}\right)$, two coefficients related to decentering distortion $\left(p_{1}, p_{2}\right)$, and two coefficients related to affinity and shear $\left(b_{1}, b_{2}\right)$. The latest were selected to account for possible image deformation occurred during the non-rigorous scanning of photo hard-copies.

The EO of all the photogrammetric blocks has been followed by the generation of 'Dense point clouds', which has been operated at 'Medium' resolution with a 'Moderate' level for depth filtering. Also in the case of these data sets, the use of top 'Quality' levels has led to a decrease of the final point cloud's completeness. On the other hand, the DEM's to be derived from point clouds should be used for the analysis of terrain elevation changes, and consequently the interest on smaller objects like buildings has been retained of secondary relevance. Then DEM's and orthoimages have been produced from 'Dense point clouds.' After the generation of the orthoimages, a refinement of the horizontal geo-referencing has been obtained with the overlay to existing vector maps. A regional raster map at scale 1:5,000 was used to this purpose. In Figure 4, the overlap between a portion of the orthophoto produced from photos dating back to 1986 and the regional raster map is shown.

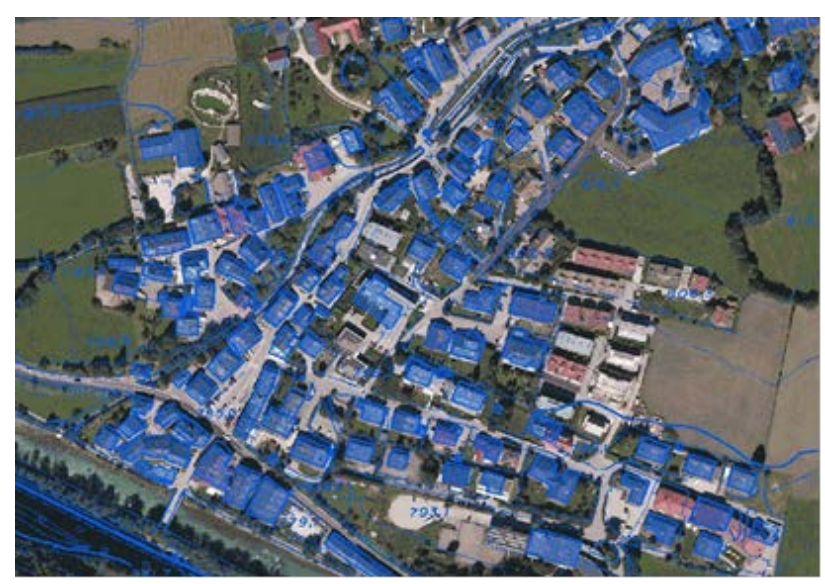

Figure 4. Overlay of the orthophotos corresponding to 1986 data set and the regional raster map at scale 1:5,000, after horizontal shift refinement.

\subsection{Analysis of the results}

Provided that the analyses listed in Subsection 2.4 may give some useful information about the quality of EO, the expected theoretical accuracy have been computed using formulas for the stereo configuration (Kraus, 2008). In Table 5 some results have been reported, which show a certain agreement with those findings obtained with APP. In this analysis, we assumed a precision of image coordinate measurement $\left(\sigma_{i m}\right)$ equal to the pixel size in the dense image matching stage. Since this task has been operated at 'Medium Quality' in APP, the pixel size (i.e., $160 \mu \mathrm{m})$ of subsampled images at corresponding level was considered as four times the one of the original digitized images.

\begin{tabular}{|c|c|c|c|c|c|}
\hline Year & $\boldsymbol{\sigma}_{\text {im }}[\boldsymbol{\mu m}]$ & $\mathbf{B}[\mathbf{m}]$ & $\boldsymbol{\sigma}_{\mathbf{z}}[\mathbf{m}]$ & $\boldsymbol{\sigma}_{\mathbf{X Y}}[\mathbf{m}]$ & GSD [m] \\
\hline 1954 & 160 & 12210 & 6.5 & 7.6 & 7.6 \\
\hline 1959 & 160 & 4120 & 6.5 & 4.4 & 4.4 \\
\hline 1986 & 160 & 5930 & 4.9 & 4.6 & 4.8 \\
\hline 1995 & 160 & 7700 & 8.6 & 7.0 & 7.2 \\
\hline
\end{tabular}

Table 5. Expected theoretical accuracy for 3D points evaluated on the basis of standard formulas for the stereo-case in aerial photogrammetry (Kraus, 2008); B is the baseline between consecutive images.

The overlay between the orthoimage time series (including nine epochs: $1954,1959,1986,1988,1994,1995,2000,2006$, 2012) and raster maps has allowed to detect those changes related to land use and building construction. In order to analyse the elevation changes, a LiDAR DTM has been used instead. This terrain model is the same adopted for deriving the elevations of GCP's. All DEM's obtained from photogrammetric processing and the LiDAR DEM have been compared in a pairwise manner using M3C2 function (Lague et al., 2013) of CloudCompare ver. 2.9 (www.cloudcompare.org). The application of this methodology avoided the need of resampling DEM's before comparing them. Since the LiDAR DTM represents the topographic surface, whilst photogrammetric DEM's are DSM's are also including the objects over the ground (buildings, trees, 
electric poles, etc.), some differences in each DoD may depend on this fact. Consequently, this analysis has been bounded to those areas made up of bare ground. In Table 6 the results on three of these regions are shown in term of average elevation differences. In general, a reduction of the absolute values of the mean differences (in the rightmost column of Table 6) may be noticed, especially in Regions 1 and 3. This finding may be mainly due to the quality of the photographic material, which is expected to be lower in the older data sets. The different photo scales may have also had influence, according to the relative flight heights reported in Table 4. In Region 2, the progressive reduction of the mean differences could be due to some ongoing excavation process. A visual analysis of the orthoimages in the corresponding periods has not confirmed this assumption. Consequently, this behaviour cannot be assigned a specific reason, but it may be due to the effect of random errors as estimated in Table 5. On the other hand, the results of the analysis on the 'Test block' reported in Table 2 also enhanced a nonhomogenous behaviour of the differences within the study area.

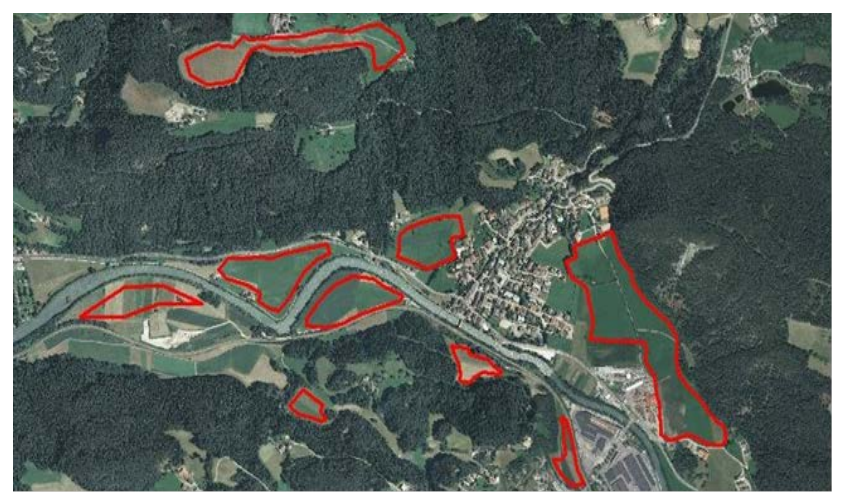

Figure 5. Regions where comparing the photogrammetric DEM's to the LiDAR DTM.

\begin{tabular}{|c|c|c|c|c|}
\hline Region & $\begin{array}{c}\text { Year of } \\
\text { DEM }\end{array}$ & $\begin{array}{c}\text { Mean height } \\
\text { of LiDAR } \\
\text { DTM [m] }\end{array}$ & $\begin{array}{c}\text { Mean } \\
\text { height of } \\
\text { DEM's [m] }\end{array}$ & $\begin{array}{c}\text { Mean difference } \\
\text { of DoD [m] }\end{array}$ \\
\hline \multirow{4}{*}{1} & 1954 & \multirow{4}{*}{798.25} & 787.45 & 10.79 \\
\hline & 1959 & & 780.08 & 18.17 \\
\hline & 1986 & & 793.74 & 4.51 \\
\hline & 1995 & & 797.40 & 0.85 \\
\hline \multirow{4}{*}{2} & 1954 & \multirow{4}{*}{788.00} & 787.64 & 0.36 \\
\hline & 1959 & & 788.68 & -0.68 \\
\hline & 1986 & & 791.24 & -3.24 \\
\hline & 1995 & & 797.88 & -9.88 \\
\hline \multirow{4}{*}{3} & 1954 & \multirow{4}{*}{1035.55} & 1031.04 & 4.51 \\
\hline & 1959 & & 1040.27 & -4.71 \\
\hline & 1986 & & 1032.62 & 2.94 \\
\hline & 1995 & & 1037.23 & -1.68 \\
\hline
\end{tabular}

Table 6. Comparison between the average heights of a reference LiDAR DTM and photogrammetric DEM's.

\subsection{Historical analysis}

After the analysis of data quality, the time series of orthoimages and DEM's have been applied to detect land changes occurred since the 50's. This analysis should try to retrieve information on the foundations of buildings along the planned axis of the new tunnel, as well as to understand hydrogeological processes that might have occurred. To this purpose, the planimetric position of buildings and other areas where relevant geological processes happened, has been drawn on the basis of the interpretation of orthoimages. In a GIS environment, some attributes have been associated to the polygons to record the construction period, the building typology, and possible geological processes. In Figure 6 the output of this study is shown, where the construction period of each building is displayed using a different colour. Provided that the construction techniques of building foundations have progressively modernized during the past decades, the hypothesis has been that the oldest buildings might have deeper foundation structures that could interfere with tunnel digging. Thus, the identification of older buildings in the nearby of the excavation areas would require thorough in-situ investigations.

Among other classified areas, the polygon ID=223 has been identified as an old open sand or gravel pit, now covered by soil and used for agricultural purpose. As it is clearly visible in the orthoimages patches shown in Figure 7, the excavation process has lasted between 1995 and 2006. This is an example of an area where the analysis of archive photos has provided unique geo-referenced information to address further in-situ investigations.

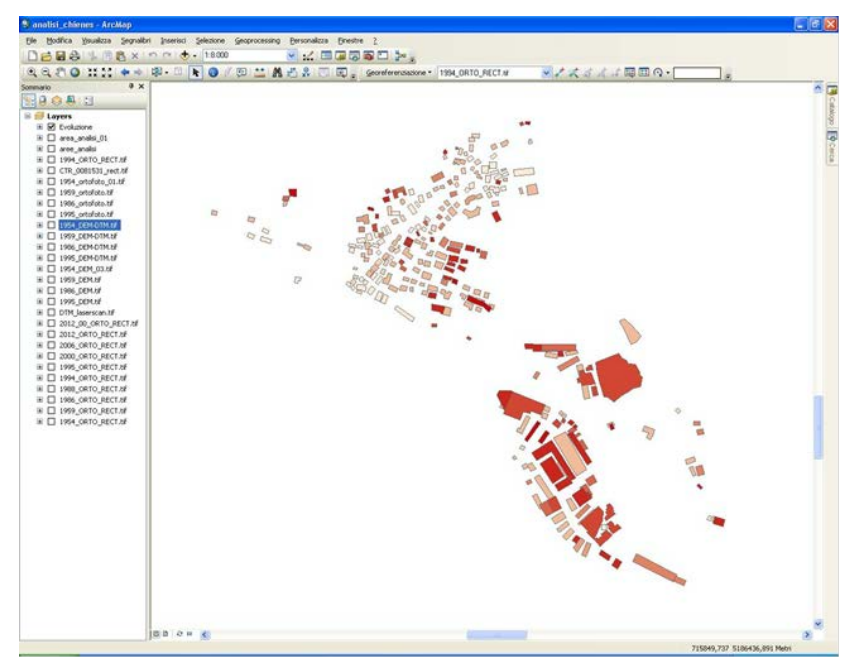

Figure 6. Classification of buildings depending on the period of construction, as well as other areas that might have been interested by relevant geological processes.

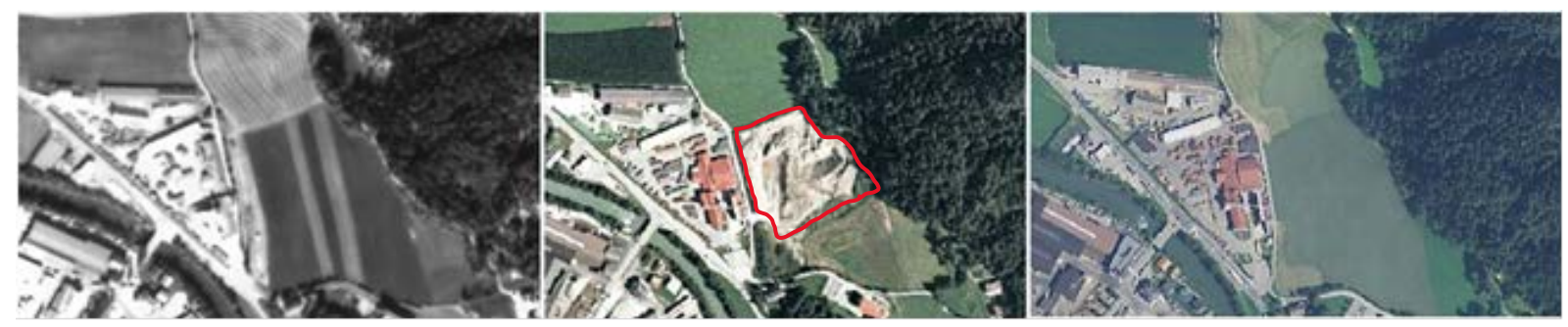

Figure 7. Patches from orthoimages (from left to right: 1986, 1995, 2006) depicting a portion of the territory of Kiens/Chienes in correspondence of polygon ID= 223 (in red on the central patch), where a covered former open pit has been found. 


\section{CONCLUSIONS}

This paper has shown how the photogrammetric processing of archive photos has the potential to offer useful geo-referenced information for the analysis of an area where an infrastructure has to be built. The study has been carried out using a popular lowcost software package (Agisoft Photoscan Professional ${ }^{\circledR}$ ver. 1.2.4) which is normally used in close-range and UASphotogrammetry applications. Here its usage with digitized archive analogue photos has been illustrated, analysed and applied through a couple of case studies (a block made up of four photos with GNSS ground control and four blocks of three photos each without precise ground control). This study has demonstrated how the adopted tool is not equivalent to the use of photogrammetric software designed for processing aerial images. One important limitation is undoubtedly due to problems when using large size imagery in the order of more than $100 \mathrm{M}$ pixels. The full potential of dense matching cannot be exploited to create a high-resolution point cloud. On the other hand, the application within a case study consisting of an area where a road tunnel had to be excavated, has produced outputs useful to define where preliminary investigations are necessary. As demonstrated in the two examples reported in this paper, the altimetric precision is a week point of the adopted procedure. A more consistent number of ground control points would help to improve the quality of final output.

Future research will concern more analysis on the use of the same and other software packages to accomplish photogrammetric processing tasks on larger blocks. Moreover, new version 1.4 of Agisoft Photoscan Professional ${ }^{\circledR}$ has been announced which will include the direct usage of aerial metric cameras with fiducial marks.

\section{ACKNOWLEDGEMENTS}

Acknowledgements go to Agisoft company for distributing 30days free licences of software Photoscan.

\section{REFERENCES}

\section{References from Books}

Hartley, R., and A. Zisserman, 2003. Multiple view geometry in computer vision, $2^{\text {nd }}$ edition. Cambridge University Press, Cambridge, UK.

Kraus, K., 1997. Photogrammetry - Vol. 2. Dümmler Verlag, Bonn, Germany.

Kraus, K., 2008. Photogrammetry - Geometry from Images and Laser Scans. Walter de Gruyter, Berlin, Germany.

Luhmann, T., Robson, S., Kyle, S., and J. Böhm, 2014. Close Range Photogrammetry: $3 D$ Imaging Techniques $-2^{\text {nd }}$ Edition. Walter De Gruyter Inc., Berlin, Germany, 684 pages.

\section{References from Journals}

Colomina, I., and P. Molina, 2014. Unmanned aerial systems for photogrammetry and remote sensing: A review. ISPRS J. Photogramm. Remote Sens., 92: 79-97.

Dowman, I.J., Ebner, H., and C. Heipke, 1992. Overview of European Developments in Digital Photogrammetric Workstations. Photogramm. Eng. Remote Sens., 58: 51-56.
Forlani, G., Roncella, R., and C. Nardinocchi, 2015. Where is Photogrammetry heading to? State of the art and trends. Rend. Fis. Acc. Lincei, 26(Supp. 1): S85-S96.

Grün, A., 2012. Development and Status of Image Matching in Photogrammetry. The Photogrammetric Record, 27(137): 36-57, DOI: $10.1111 /$ j.1477-9730.2011.00671.x

Kim, K., Jezek, K. C., and H. Liu, 2007. Orthorectified image mosaic of Antarctica from 1963 Argon satellite photography: image processing and glaciological applications. Int. J. Remote Sens., 28(23): 5357-5373.

Kunz, M., King, M.A., Mills, J.P., Miller, P.E., Fox, A.J., Vaughan, D.G., and S.H. Marsh, 2012. Multi-decadal glacier surface lowering in the Antarctic Peninsula”, Geophysical Research Letters, 39, paper No. L19502, 5 pages.

Lague, D., Brodu, N., and J. Leroux, 2013. Accurate 3D comparison of complex topography with terrestrial laser scanner: application to the Rangitikei canyon (N-Z). ISPRS J. Photogramm. Remote Sens., 82: 10-26.

Li, R., Ye, W., Qiao, G., Tong, X., Liu, S., Kong, F., and X. Ma, 2017. A New Analytical Method for Estimating Antarctic Ice Flow in the 1960s from Historical Optical Satellite Imagery. IEEE Trans. Geosci. Remote Sens., 55(5): 2771-2785.

Lindenbergh, R., and P. Pietrzyk, 2015. Change detection and deformation analysis using static and mobile laser scanning. Applied Geomatics, 7, 65-74.

Luhmann, T., Fraser, C., and H.-G. Maas, 2016. Sensor modelling and camera calibration for close-range photogrammetry. ISPRS J. Photogramm. Remote Sens., 115: 3746.

Toth, C., and G. Jozkow, 2016. Remote sensing platforms and sensors: A survey. ISPRS J. Photogramm. Remote Sens., 115: 2236.

Walstra, J., Dixon, N., Chandler, J.H., 2007. Historical aerial photographs for landslide assessment: two case histories. Quart. J. Eng. Geol. Hydrogeol., 40. 315-332.

\section{References from other literature}

Ackermann, F., 1991. Structural changes in photogrammetry. In: Proc of " $43^{\text {rd }}$ Photogrammetric Week," 9-14 Sept, Institute of Photogrammetry, University of Stuttgart, Germany, pp. 1-15.

Baltsavias, E.P., 1994. Test and calibration procedures for image scanners. Int. Arch. Photogramm. Remote Sens. Spatial Inf. Sci., Vol. 30, Part 1, pp. 163-170.

Barazzetti, L., Forlani, G., Remondino, F., Roncella, R., and M. Scaioni M., 2011. Experiences and achievements in automated image sequence orientation for close-range photogrammetric projects. Proc. of SPIE, Vol. 8085, paper No. 80850F, 13 pp. (e-doc), DOI: 10.1117/12.890116.

Barazzetti, L., Mussio, L., Remondino, F., and M. Scaioni, 2011. Targetless camera calibration. Int. Arch. Photogramm. Remote Sens. Spatial Inf. Sci., Vol. 38, Part 5/W16, 8 pages.

Previtali, M., Barazzetti, L., and M. Scaioni, 2014. Accurate 3D surface measurement of mountain slopes through a fully automated imaged-based technique. Earth Sci. Inf., 7(2): 109122, DOI: 10.1007/s12145-014-0158-2.

Verhoeven, G., Karel, W., Stuhec, S., Doneus, M., Trinks, I., and N. Pfeifer, 2015. Mind your grey tones - examining the influence of decolourization methods on interest point extraction and matching for architectural image-based modelling. Int. Arch. Photogramm. Remote Sens. Spatial Inf. Sci., Vol. 40, 307-314. 